\title{
THE COMPLEXITY OF CHECKING IDENTITIES OVER FINITE GROUPS
}

\author{
GÁBOR HORVÁTH AND CSABA SZABÓ
}

\begin{abstract}
We analyze the computational complexity of solving a single equation and checking identities over finite meta-abelian groups. Among others we answer a question of Goldmann and Russel from '98: We prove that it is decidable in polynomial time whether or not an equation over the six element group $S_{3}$ has a solution.
\end{abstract}

\section{INTRODUCTION}

The computational complexity of the word problem in algebra is of greater and greater interest. In this paper we present results about the computational complexity of checking identities over finite groups. We use standard notations in computational complexity (see [3]).

In 1997 Ross Willard gave a talk at The Fields Institute where he presented several results and problems concerning algebraic complexity questions about rings. He defined two versions of the word problem. There are two kinds of words. A term on an algebra $\mathbf{A}$ is an expression that can be obtained using (iterated) compositions of the basic operations and projections. Projections are trivial operations satisfying $p_{i}^{n}\left(x_{1}, \ldots, x_{n}\right)=x_{i}$. A polynomial on an algebra $\mathbf{A}$ is an expression that can be obtained using (iterated) compositions of the basic operations, projections and nullary, constant operations. The two versions of the word problem are the term equivalence (TERM-EQ), and the polynomial equivalence (POL-EQ) problems.

Definition 1. Let $\mathbf{A}$ be an algebra. Two terms (polynomials) $t_{1}$ and $t_{2}$ are called equivalent $\left(t_{1}\left(x_{1}, \ldots, x_{n}\right) \equiv t_{2}\left(x_{1}, \ldots, x_{n}\right)\right.$ or shortly $t_{1} \equiv$ $t_{2}$ ) if the values of the two terms (polynomials) are equal at every substitution from A. An instance of the term (polynomial) equivalence problem (TERM-EQ A, POL-EQ $\mathbf{A}$ is a pair of terms (polynomials) $t_{1}$ and $t_{2}$ with the question whether or not the two terms (polynomials) are equivalent.

For finite structures there is an obvious algorithm to decide these problems. Indeed, one can check every possible substitution, and if the 
two terms (polynomials) agree at all of them then they are equivalent. On the other hand, if one finds a tuple of elements NOT satisfying the equation, then it can be showed in polynomial time that the two words are not equivalent. Hence for finite algebras both equivalence problems are obviously in coNP. In what follows, all algebras will be finite.

Thus two terms, $t_{1}$ and $t_{2}$ are equivalent if and only if $t_{1}=t_{2}$ is an identity over $\mathbf{A}$. In case $\mathbf{A}$ is a group, this is equivalent to $t_{1} t_{2}^{-1} \equiv 1$. Hence, we introduce the following definition, often used in group theory.

Definition 2. A term over a group is called an identity if it is equivalent to 1 , the identity element of the group.

Willard in his talk discussed these two problems for rings. It was already known ([5]) that for a commutative ring $\mathbf{R}$ the TERM-EQ problem is in $\mathrm{P}$ if $\mathbf{R}$ is nilpotent and coNP-complete otherwise. Burris and Lawrence proved in [2] that the same holds for rings in general. Following their proof it is easy to see that for a nilpotent ring $\mathbf{R}$ the problem POL-EQ $\mathbf{R}$ is in $\mathrm{P}$ and it is a straightforward consequence of their result that if the ring is not nilpotent, then POL-EQ $\mathbf{R}$ is coNPcomplete.

For groups the answer is far less complete. An unpublished result of Burris and Lawrence (1994) is the following.

Theorem 3. Let $\mathbf{G}$ be a group. If $\mathbf{G}$ is nilpotent, then TERM-EQ $\mathbf{G}$ is in P. If $\mathbf{G}$ is not solvable, then TERM-EQ $\mathbf{G}$ is coNP-complete.

In this paper we would like to extend these results for a class of solvable non-nilpotent groups. We prove that several kinds of semidirect products admit polynomial time solvable TERM-EQ problem. For example we prove that checking identities is easy for the dihedral groups, for the alternating group $A_{4}$, for the wreath product of two cyclic groups, etc.

The other problem to investigate is the complexity of solving equations and systems of equations over finite algebras. These problems arise from unification theory ([6]), formal languages ([11]) and, naturally, from universal algebra.

Definition 4. Let $\mathbf{A}$ be an algebra. The input of the polynomial satisfiability problem (POL-SAT A) is a pair of polynomials $s$ and $t$ with the question whether there is a substitution of the variables from A such that the values of the two polynomials are the same.

Definition 5. Let $\mathbf{A}$ be an algebra. The input of the polynomial system-satisfiability problem (POL-SYS A) are $2 n$ polynomials $s_{1}, \ldots, s_{n}$ and $t_{1}, \ldots, t_{n}$ with the question whether there is a substitution of the variables from $\mathbf{A}$ such that $s_{i}=t_{i}$ for all $i=1, \ldots, n$. 
THE COMPLEXITY OF CHECKING IDENTITIES OVER FINITE GROUPS

3 [8]:

The complexity POL-SYS is fully characterized for groups in [4] and

Theorem 6. Let $\mathbf{A}$ be a group. The problem POL-SYS A is in $P$ if $\mathbf{A}$ is Abelian and it is NP-complete otherwise.

The characterization of solving a single equation looks more complicated, though $([4])$.

Theorem 7. Let $\mathbf{G}$ be a group. If $\mathbf{G}$ is nilpotent, then POL-SAT $\mathbf{G}$ is in P. If $\mathbf{G}$ is not solvable, then POL-SAT $\mathbf{G}$ is coNP-complete.

The result tells nothing about non-nilpotent solvable groups. Goldmann and Russel explicitly ask in [4] to decide the complexity of solving an equation over $S_{3}$.

The POL-SAT problem was first examined for monoids and semigroups. Klíma [7] has analyzed the question for semigroups of size at most 6 . He proved for almost all of these semigroups that solving an equation is in either in $\mathrm{P}$ or NP-complete. The only remaining case is the 6 element "monoid" $S_{3}$. He conjectures that the problem is in P.

In this paper we show the following: If $\mathbf{G} \simeq \mathbf{A} \rtimes \mathbf{B}$, where $\mathbf{A} \simeq \mathbf{Z}_{\mathbf{p}}$ and $\mathbf{B} \simeq \mathbf{Z}_{\mathbf{q}}$ for some primes $p$ and $q$, then POL-SAT $\mathbf{G}$ is in P. Thus, with $\mathbf{Z}_{\mathbf{3}} \simeq \mathbf{A}$ and $\mathbf{Z}_{\mathbf{2}} \simeq \mathbf{B}$ we answer the questions of both Goldmann and Russel and Klíma.

The result suggests that the complexity of TERM-EQ and POL-SAT for a finite algebra $\mathbf{A}$ is always the same. This is far from to be true. Seif and Szabó present a 10 element semigroup (see [10]) for which the term-equivalence problem is decidable in polynomial time and the POL-SAT problem is coNP-complete. An even stronger result of Klíma is the following (see [7]):

Theorem 8. There is a semigroup $\mathbf{S}$ of size 24 such that POL-SAT $\mathbf{S}$ is NP-complete and POL-EQ $\mathbf{S}$ is in P.

It may happen, though, that the complexity of the two problems coincide in case of groups. At this point we do not even know the answer for $\mathbf{S}_{4}$.

\section{SEMIDIRECT PRODUCTS}

In this section we will prove for a class of non-nilpotent groups that the POL-EQ problem (so the TERM-EQ problem also) can be solved in polynomial time. The group operation will always be multiplication. The identity element of a group will be denoted by 1 . The following method will play a crucial role in our investigation. 
Collecting procedure: Let $\mathbf{G} \simeq \mathbf{A} \rtimes \mathbf{B}$ where $\mathbf{A}$ is Abelian and let $t=x_{1} x_{2} \ldots x_{k}$ be a group polynomial over $\mathbf{G}$. Without loss of generality we assume that the $x_{i}$ are constants or variables over $\mathbf{G}$. Every element of $\mathbf{G}$ can be uniquely written of the form $b a$ where $a \in \mathbf{A}$ and $b \in \mathbf{B}$. So we write $x_{i}$ of the form $b_{i} a_{i}$ where $a_{i} \in \mathbf{A}$ and $b_{i} \in \mathbf{B}$. Collecting the elements of $\mathbf{B}$ to the left we obtain

$$
t=\left(b_{1} b_{2} \ldots b_{k}\right) \cdot\left(a_{1}^{b_{2} b_{3} \ldots b_{k}} a_{2}^{b_{3} \ldots b_{k}} \ldots a_{k-1}^{b_{k}} a_{k}\right) .
$$

This term is an identity if and only if both

$$
b_{1} b_{2} \ldots b_{k}
$$

and

$$
\left(a_{1}^{b_{2} b_{3} \ldots b_{k}} a_{2}^{b_{3} \ldots b_{k}} \ldots a_{k-1}^{b_{k}} a_{k}\right)
$$

are identities (i.e. both are identically 1 for all substitutions over $\mathbf{G}$ ). Let us examine the latter expression. Substitute $a_{i}=1$ for all $i$, where $x_{i}$ was a variable, not constant. Then we get $t^{\prime}=c_{1}^{w_{1}} c_{2}^{w_{2}} \ldots c_{m}^{w_{m}}$, where all $c_{i}$ s are constants from $\mathbf{A}$ and $w_{i}$ is a word over $\mathbf{B}$ (let us call $t^{\prime}$ the constant part of (2)). Let us fix $j$. Substituting $a_{i}=1$ for $i \neq j$ (where $a_{i}$ is not constant) we obtain an identity of the form $t_{j}^{\prime} t^{\prime}$ where $t_{j}^{\prime}=a_{j}^{h_{1}} a_{j}^{h_{2}} \ldots a_{j}^{h_{l}}$ and $l$ is the number of the occurrences of $x_{j}$ in $t$ and $h_{i}$ is a semigroup polynomial over $\mathbf{B}$ for every $1 \leq i \leq l$. Obviously, (2) is an identity if and only if $t^{\prime}$ and $t_{j}^{\prime}$ are identities for every $1 \leq j \leq k$. Hence we are looking for the complexity of checking whether or not $b_{1} b_{2} \ldots b_{k}, t$ and $t_{j}^{\prime}$ are all identities.

Lemma 9. Let $\mathbf{F}$ be a field of prime characteristic $p$ and $\mathbf{H} \leq \mathbf{F}^{*}$. For a polynomial $f(\bar{x}) \in \mathbf{F}\left[x_{1}, x_{2}, \ldots, x_{k}\right]$ it can be checked in polynomial time whether or not it vanishes on $\mathbf{H}$.

Proof. Let $a$ be a generator of $\mathbf{F}^{*}$ and let $\mathbf{H}=\left\langle a^{t}\right\rangle$. Putting $z_{j}=x_{j}^{t}$ we have $f(\bar{x})$ is identically 0 over $\mathbf{H}$ if and only if $f(\bar{z})$ is identically 0 over $\mathbf{F}^{*}$. A polynomial $g \in \mathbf{F}\left[x_{1}, \ldots, x_{k}\right]$ admits this latter property if and only if $g=\sum\left(x_{i}^{q-1}-1\right) g_{i}(\bar{x})$ for some $g_{i} \in \mathbf{F}\left[x_{1}, \ldots, x_{k}\right]$, where $|\mathbf{F}|=q$. This condition can be checked in linear time since we only need to divide $g$ by $x_{i}^{q-1}-1$ (i.e. substitute $x_{i}^{q-1}=1$ ) for all $i \in\{1, \ldots, k\}$ and the remaining expression has to be 0 .

Theorem 10. If $\mathbf{G} \simeq \mathbf{A} \rtimes \mathbf{B}$ where $\mathbf{A} \simeq \mathbf{Z}_{\mathbf{p}}$ for some prime $p$, and POL-EQ $\mathbf{B}$ is in $P$ then POL-EQ $\mathbf{G}$ is in $P$. 
Proof. The subgroup $\mathbf{B}$ acts on $\mathbf{A}$. Now, Aut $(\mathbf{A}) \simeq \mathbf{C}_{p-1}$, the cyclic group of order $p-1$ and consists of the maps $a \rightarrow a^{l}$ for every $a \in \mathbf{A}$ for some $1 \leq l \leq p-1$. Thus there is a homomorphism $\phi: \mathbf{B} \rightarrow$ $\mathbf{C}_{p-1}$ such that $a^{b}=a^{\phi(b)}$ for every $a \in \mathbf{A}$. Now, using the collecting procedure it is enough to check whether or not $b_{1} b_{2} \ldots b_{k}, a_{j}^{h_{1}} a_{j}^{h_{2}} \ldots a_{j}^{h_{l}}$ and $c_{1}^{w_{1}} c_{2}^{w_{2}} \ldots c_{m}^{w_{m}}$ are identities. The first condition can be checked in polynomial time by the assumption. For the second one we rewrite the expression $a_{j}^{h_{1}} a_{j}^{h_{2}} \ldots a_{j}^{h_{l}}=a_{j}^{\phi\left(h_{1}\right)} a_{j}^{\phi\left(h_{2}\right)} \ldots a_{j}^{\phi\left(h_{l}\right)}=a_{j}^{w_{1}+w_{2}+\cdots+w_{l}}$. Here $w_{j}$ denotes the image of $h_{j}$ at $\phi$. Substituting $\phi\left(b_{j}\right)=y_{j}$ we have $w_{j}$ as a product of some of $y_{1}, \ldots y_{k}$ over $\mathbf{Z}_{\mathbf{p}}$, shortly a monomial, and $f=w_{1}+w_{2}+\cdots+w_{l}$ is a $k$-variable polynomial over $\phi(\mathbf{B})$ where both the addition and the multiplication is understood in $\mathbf{Z}_{\mathbf{p}}$. The expression $a_{j}^{w_{1}+w_{2}+\cdots+w_{l}}$ is an identity if and only if $f$ attains 0 every time when we substitute elements of $\phi(\mathbf{B})$ for the variables. And this can be checked in polynomial time by Lemma 9 . Finally, $c_{1}^{w_{1}} c_{2}^{w_{2}} \ldots c_{m}^{w_{m}}$ can be written in the form $c^{w_{1}^{\prime}} c^{w_{2}^{\prime}} \ldots c^{w_{m}^{\prime}}$, where $c$ is the generator, of $\mathbf{A}$. Using the same idea, this is an identity if and only if $w_{1}^{\prime}+\cdots+w_{m}^{\prime}$ attains 0 every time when we substitute elements of $\phi(\mathbf{B})$ for the variables. And this can be checked in polynomial time by Lemma 9, again.

Corollary 11. If $\mathbf{G} \simeq \mathbf{A} \rtimes \mathbf{B}$, where $\mathrm{POL}-\mathrm{EQ} \mathbf{B}$ is in $P$, and $\mathbf{A} \simeq \mathbf{Z}_{\mathbf{m}}$ where $m$ is squarefree, then POL-EQ $\mathbf{G}$ is in $P$.

Proof. Now, $\mathbf{A} \simeq \oplus_{p \mid m} \mathbf{Z}_{\mathbf{p}}$ and all summands are $\mathbf{B}$ invariant. Every constant can be uniquely decomposed into a product of elements from $\mathbf{Z}_{\mathbf{p}}$ for $p \mid m$. For a polynomial $p$ let $t_{(p)}$ denote the polynomial when we replace each constant by its $p$ part. Obviously, a polynomial is an identity over $\mathbf{G}$ if and only if $t_{(p)}$ is an identity over $\mathbf{Z}_{\mathbf{p}} \rtimes \mathbf{B}$ for every prime $p$ dividing $m$. This can be checked in polynomial time by Theorem 10.

Unfortunately the same idea does not work for a noncyclic normal subgroup, A. The collecting procedure can be used in a few other cases, though.

Theorem 12. Let $\mathbf{G} \simeq \mathbf{A} \rtimes \mathbf{B}$ such that the following hold:

(a) $\mathbf{A}$ is Abelian and the exponent of $\mathbf{A}$ is squarefree;

(b) POL-EQ B is in P;

(c) for ever prime $p$ dividing the size of $\mathbf{A}$ and $\mathbf{P} \in S y l_{p}(\mathbf{A})$ the group $\mathbf{B} / C_{\mathbf{B}}(\mathbf{P})$ is Abelian and $p \nmid\left|\mathbf{B} / C_{\mathbf{B}}(\mathbf{P})\right|$, where $C_{\mathbf{B}}(\mathbf{P})$ denotes the centralizer of $\mathbf{P}$ in $\mathbf{B}$.

Then POL-EQ G is in P. 
Proof. After the collection procedure we see that it is enough to check identities over B and identities of the form (2)

$$
a^{x_{1}^{k_{11}} x_{2}^{k_{12}} \ldots x_{n}^{k_{1 n}}} a^{x_{1}^{k_{21}}} x_{2}^{k_{22}} \ldots x_{n}^{k_{2 n}} \ldots a^{x_{1}^{k_{l 1}} x_{2}^{k_{l 2}} \ldots x_{n}^{k_{l n}}}
$$

and $c_{1}^{w_{1}} c_{2}^{w_{2}} \ldots c_{m}^{w_{m}}$ for the constants. The Sylow subgroups of $\mathbf{A}$ are $\mathbf{B}$ invariant, hence it is enough to check the identity for the Sylows of $\mathbf{A}$. Thus we may assume that $\mathbf{A}$ is an elementary Abelian $p$-group. Let $\mathbf{A} \simeq \mathbf{Z}_{p}^{m}$ and let $\varphi: \mathbf{B} \rightarrow$ Aut $\mathbf{Z}_{p}^{m} \simeq G L_{m}\left(\mathbf{Z}_{p}\right)$ be the action of $\mathbf{B}$ on $\mathbf{A}, \varphi(\mathbf{B})=\mathbf{H}$. With these notations we need to check identity (2) for $\mathbf{G} \simeq \mathbf{Z}_{p}^{m} \rtimes \mathbf{H}$, where $\mathbf{H}$ is an Abelian matrix group acting faithfully on $\mathbf{Z}_{p}^{m}$ (note that $\mathbf{H} \simeq \mathbf{B} / C_{\mathbf{B}}\left(\mathbf{Z}_{p}^{m}\right)$ ). Let $\mathbf{S}$ denote the subring of the ring of $m$ by $m$ matrices generated by $\mathbf{H}$. Now (3) can be rewritten as:

$$
a^{x_{1}^{k_{11}}} x_{2}^{k_{12} \ldots x_{n}^{k_{1 n}}+x_{1}^{k_{21}}} x_{2}^{k_{22} \ldots x_{n}^{k_{2 n}}+\cdots+x_{1}^{k_{l 1}}} x_{2}^{k_{l 2}} \ldots x_{n}^{k_{l n}}
$$

and it is enough to check whether or not the exponent

$$
x_{1}^{k_{11}} x_{2}^{k_{12}} \ldots x_{n}^{k_{1 n}}+x_{1}^{k_{21}} x_{2}^{k_{22}} \ldots x_{n}^{k_{2 n}}+\cdots+x_{1}^{k_{l 1}} x_{2}^{k_{l 2}} \ldots x_{n}^{k_{l n}}
$$

is identically 0 in $\mathbf{S}$ when substituting the elements of $\mathbf{H}$. The ring $\mathbf{S}$ acts semisimply on $\mathbf{Z}_{p}^{m}$, because $p \nmid|\mathbf{H}|$. By Maschke's theorem $\mathbf{S}$ is a direct sum of matrix-rings. As $\mathbf{H}$ is commutative, $\mathbf{S}$ is commutative, as well, hence $\mathbf{S}$ is a direct sum of fields: $\mathbf{S}=\oplus_{i=1}^{s} \mathbf{F}_{q_{i}}$. Thus $\mathbf{H} \leq \mathbf{S}^{*} \simeq$ $\oplus_{i=1}^{s} \mathbf{F}_{q_{i}}^{*}$. Let $\mathbf{H}_{i}$ denote the projection of $\mathbf{H}$ to its $i$-th coordinate. Expression (5) is identically 0 over $\mathbf{S}$ if and only if it is 0 at every substitution from $\mathbf{H}_{i}$ for every $i \leq s$. By Lemma 9 this can be checked in polynomial time, and so POL-EQ $\mathrm{G}$ is in $\mathrm{P}$.

Finally, consider the identity $c_{1}^{w_{1}} c_{2}^{w_{2}} \ldots c_{l}^{w_{m}}=1$. Here we can write every $c_{j}$ as a linear combination of some fixed basis, $\left\{v_{i}\right\}$, of $\mathbf{A}$. Let $c_{j}=$ $\prod v_{i}^{\lambda_{j i}}$. Thus, it is enough to check, whether $v_{i}^{\lambda_{1 i} w_{1}} v_{i}^{\lambda_{2 i} w_{2}} \ldots v_{i}^{\lambda_{l i} w_{l}}=1$ is an identity for all $1 \leq i \leq s$. The exponent has to be identically 0 over $\mathbf{H}_{\mathbf{i}}$, and this can be checked in polynomial time by Lemma 9 .

Corollary 13. Let $\mathbf{G} \simeq \mathbf{A} \rtimes \mathbf{B}$, where $\mathbf{A}$ and $\mathbf{B}$ are Abelian groups, such that the exponent of $A$ is squarefree and $(|\mathbf{A}|,|\mathbf{B}|)=1$ then POL-EQ G is in P.

Proof. The conditions of Theorem 12 trivially hold. 
Now, we investigate the case when neither the size nor the exponent of the normal subgroup is squarefree. The modification of the Lemma 9 remains valid for cyclic groups.

Lemma 14. Let $f\left(x_{1}, \ldots, x_{k}\right)=w_{1}+\cdots+w_{l}$ be a sum of monomials in $k$ variables over $\mathbf{Z}_{p^{\alpha}}(p>2)$ and let $\mathbf{H}$ be the $p-1$ element subgroup of $\mathbf{Z}_{p^{\alpha}}^{*}$. Then, for any $\mathbf{M} \leq \mathbf{H}$ it can be checked in polynomial time whether or not $f$ vanishes on $\mathbf{M}$.

Proof. Let $a$ be a generator of $\mathbf{H}$ and let $\mathbf{M}=\left\langle a^{t}\right\rangle$. Putting $z_{j}=x_{j}^{t}$ we have $f(\bar{x})$ is identically 0 over $\mathbf{M}$ if and only if $f(\bar{z})$ is identically 0 over $\mathbf{H}$. We claim that a polynomial $f \in Z_{p^{n}}\left[x_{1}, \ldots, x_{k}\right]$ admits this latter property if and only if $f=\sum\left(x_{i}^{p-1}-1\right) g_{i}(\bar{x})$ for some $g_{i} \in$ $Z_{p^{n}}\left[x_{1}, \ldots, x_{k}\right]$. This condition can be checked in linear time. Since the exponent of $\mathbf{H}$ is $p-1$, if $f$ is of the required form, it vanishes over $\mathbf{H}$. On the other hand, as the elements of $\mathbf{H}$ are pairwise incongruent $\bmod p$ (not only $\bmod p^{\alpha}$ ), the polynomial has to vanish over $\mathbf{Z}_{p}^{*}$, as well. By Lemma 9 this happens if and only if $f=\sum\left(x_{i}^{p-1}-1\right) g_{i 1}(\bar{x})$ $\bmod p$ and so $f=\sum\left(x_{i}^{p-1}-1\right) g_{i 1}(\bar{x})+p f_{1} \bmod p^{\alpha}$. Hence $f_{1}$ is vanishing $\bmod p^{\alpha-1}$. By the previous arguments $f_{1}=\sum\left(x_{i}^{p-1}-\right.$ 1) $g_{i 2}(\bar{x}) \bmod p$. Continuing in the same fashion we obtain that $f=$ $\sum\left(x_{i}^{p-1}-1\right) g_{i}(\bar{x})$.

The following theorem is a generalization of Theorem 10:

Theorem 15. Let $\mathbf{G} \simeq \mathbf{A} \rtimes \mathbf{B}$ such that the following hold:

(a) A is cyclic;

(b) POL-EQB is in P;

(c) for ever prime $p$ dividing the size of $\mathbf{A}$ and $\mathbf{P} \in \operatorname{Syl}_{p}(\mathbf{A})$ we have $p \nmid\left|\mathbf{B} / C_{\mathbf{B}}(\mathbf{P})\right|$.

Then POL-EQ G is in $P$.

Proof. Going along the lines of Theorem 12, we may assume that $\mathbf{A} \simeq$ $\mathbf{Z}_{p^{m}}$. Moreover, after the collection procedure, it is enough to check identities over $\mathbf{B}$ and identities of the form $f=w_{1}+w_{2}+\cdots+w_{l}=0$ over $\mathbf{B} / C_{\mathbf{B}}(\mathbf{P})$ (Note that this works for the constant part, as well, since we can write every constant as a power of the generator of $\mathbf{A}$ ). As $\mathbf{B} / C_{\mathbf{B}}(\mathbf{P}) \leq \operatorname{Aut}\left(\mathbf{Z}_{p^{\alpha}}\right)$, condition (c) implies that $\mathbf{B} / C_{\mathbf{B}}(\mathbf{P}) \leq \mathbf{H}$, where $\mathbf{H}$ denotes the $p-1$ element subgroup of $\operatorname{Aut}\left(\mathbf{Z}_{p^{\alpha}}\right)$. If $p=2$ then $\mathbf{H}=1$, if $p>2$, then identities can be checked in polynomial time over $\mathbf{B}$ and $\mathbf{H}$, by condition (b), and by Lemma 14, respectively. 


\section{SAtisfiability}

A modification of the collecting procedure and Lemma 9 will also help us to find out the complexity of the POL-SAT problem for some metacyclic groups, including $S_{3}$.

Theorem 16. For any group $\mathbf{G}$ of order $p q$ where $p$ and $q$ are primes POL-SAT G is in P.

Proof. Consider the case when $\mathbf{G} \simeq \mathbf{A} \rtimes \mathbf{B}$ where $\mathbf{A} \simeq \mathbf{Z}_{\mathbf{p}}$ and $\mathbf{B} \simeq \mathbf{Z}_{q}$. We may assume that $\mathbf{G}$ is not abelian, and so $p \neq q$.

Let $\{t, s\}$ be an instance of POL-SAT G. We would like to know whether or not $t=s$ has a solution. Multiplying by $s^{-1}$ and writing $t$ for $t s^{-1}$, we have to solve $t=1$. After the collecting procedure we obtain the following equation:

$$
t\left(g_{1} \ldots g_{k}\right)=\left(b_{1} b_{2} \ldots b_{k}\right) \cdot\left(a_{1}^{b_{2} b_{3} \ldots b_{k}} a_{2}^{b_{3} \ldots b_{k}} \ldots a_{k-1}^{b_{k}} a_{k}\right)=1 .
$$

As $p$ and $q$ are coprime, both

$$
b_{1} b_{2} \ldots b_{k}=1
$$

and

$$
a_{1}^{b_{2} b_{3} \ldots b_{k}} a_{2}^{b_{3} \ldots b_{k}} \ldots a_{k-1}^{b_{k}} a_{k}=1 .
$$

must hold. Since $\mathbf{B}$ is cyclic, we can solve $b_{1} \ldots b_{k}=1$ as a congruence $\bmod q$, and we can express one of the variables (say, $b_{1}$ ) using the other variables and constants: $b_{1}=c \prod b_{i}^{k_{i} d}$, this is what a solution looks like $\bmod q$. Substituting this expression for $b_{1}$ in $t_{1}^{\prime} t_{2}^{\prime} \ldots t_{k}^{\prime} t^{\prime}=1$, we only need to check the complexity of the satisfiability of this latter equation under the constraint for $b_{1}$. By a similar argument as in the proof of Theorem 10 we arrive at the solubility of

$$
a^{x_{1}^{k_{11}}} x_{2}^{k_{12}} \ldots x_{n}^{k_{1 n}}+x_{1}^{k_{21}} x_{2}^{k_{22}} \ldots x_{n}^{k_{2 n}}+\cdots+x_{1}^{k_{l 1}} x_{2}^{k_{l 2}} \ldots x_{n}^{k_{l n}}=1,
$$

where $a$ is a generator of $\mathbf{A}$. Now, it is enough to check whether or not the exponent attains 0 , that is whether or not

$$
x_{1}^{k_{11}} x_{2}^{k_{12}} \ldots x_{n}^{k_{1 n}}+x_{1}^{k_{21}} x_{2}^{k_{22}} \ldots x_{n}^{k_{2 n}}+\cdots+x_{1}^{k_{l 1}} x_{2}^{k_{l 2}} \ldots x_{n}^{k_{l n}}=0
$$

has a solution over $\mathbf{Z}_{p}$. As $p$ is a prime, this equation has no solution if and only if

$$
\left(x_{1}^{k_{11}} x_{2}^{k_{12}} \ldots x_{n}^{k_{1 n}}+x_{1}^{k_{21}} x_{2}^{k_{22}} \ldots x_{n}^{k_{2 n}}+\cdots+x_{1}^{k_{l 1}} x_{2}^{k_{l 2}} \ldots x_{n}^{k_{l n}}\right)^{p-1}=1
$$

is an identity. This can be checked in polynomial time by Lemma 9 , hence POL-SAT G is in P. 


\section{Problems}

Klíma's example mentioned in the introduction suggests the following question:

Problem 1. Is there an algebra $\mathbf{A}$ such that POL-EQ $\mathbf{A}$ is hard and POL-SAT $\mathbf{A}$ is in P?

If there is an example, it will not be a group. Indeed, for a group G every instance $f_{1} \equiv f_{2}$ of POL-EQ $\mathbf{G}$ can be rewritten in the form $f_{1} f_{2}^{-1} \equiv 1$. If you can check the solubility of $p=a$ in polynomial time, then the only thing to do is to check the solubility of $f_{1} f_{2}^{-1}=g$ for every $g \neq 1$. The two polynomials are equivalent if and only if none of these equations have a solution.

The smallest group not discussed in this paper is $\mathbf{S}_{4}$. This group can be considered as a semidirect product of $\mathbf{Z}_{2}^{2}$ and $\mathbf{S}_{3}$. Here, the exponent of the first group is squarefree, TERM-EQ $\mathbf{S}_{3}$ is in $\mathrm{P}$, but the action of $\mathbf{S}_{3}$ is not Abelian. If we attack this problem using our technics, then after the collecting procedure, going along the lines of the proof of Theorem 12 or Theorem 15, we should discuss terms over $M_{2}\left(\mathbf{Z}_{2}\right)$ evaluated on the invertible elements.

Problem 2. Find the complexity of TERM-EQ $\mathbf{S}_{4}$ and POL-SAT $\mathbf{S}_{4}$.

\section{REFERENCES}

[1] D. M. Barrington, P. McKenzie, C. Moore, P. Tesson, D. Thérien, Equation satisfiability and program satisfiability for finite monoids, Math. Found. Comp. Sci. (2000, Bratislava), 127-181.

[2] S. Burris, J. Lawrence, The equivalence problem for finite rings, Journal of Symbolic Computation 15 (1993), 67-71.

[3] M. R. Garey, D. S. Johnson, "Computers and intractability", W.H.Freeman \& Co., San Francisco, 1979.

[4] M. Goldmann, A. Russell, The complexity of solving equations over finite groups, Proceedings of the Fourteenth Annual IEEE Conference on Computational Complexity, Atlanta, Georgia, May, 1999, 80-86

[5] H. Hunt, R. Stearns, The complexity for equivalence for commutative rings, Journal of Symbolic Computation 10 (1990), 411-436

[6] O. Klíma, Complexity of unification and matching problems in the varieties of idempotent semigroups, Int. J. of Algebra and Comp., to appear

[7] O. Klíma, Complexity issues of checking identities in finite monoids, manuscript

[8] B. Larose, L. Zádori, Taylor terms, constraint satisfaction and the complexity of polynomial equations over finite algebras, Int. J. of Alg. and Comp., submitted (2004)

[9] J. Lawrence, R. Willard, The complexity of solving polynomial equations over finite rings, manuscript (1997) 
[10] S. Seif and Cs. Szabó, The computational complexity of checking identites in simple semigroups and matrix semigroups over finite fields, Semigroup Forum (2002)

[11] P. Tesson, D. Thérien, Complete classifications for the communication complexity of regular languages, STACS '03

E-mail address: ghorvath@cs.elte.hu

E-mail address: csaba@cs.elte.hu

EÖtvös Loránd University, Department of Algebra and Number Theory, 1117 Budapest, PÁzmány PÉter sétány 1/C, Hungary 